\title{
在水相中钯催化氧化偶氮苯的区域选择性邻位酰基化反应
}

\author{
陈晓培*,† 马志伟 ${ }^{\dagger}$ 王川川 刘俊桃 吴金松 \\ (河南牧业经济学院理学部 郑州 450046)
}

\begin{abstract}
摘要 以醇为酰基化试剂, 在水溶液条件下, 发展了一种简便、高效的钯催化氧化偶氮苯酰基化反应体系. 在此体系 中，醇被氧化为醛，实现氧化偶氮苯的邻位酰基化反应，具有很好的区域选择性，以中等到较高收率得到酰基化的氧 化偶氮苯衍生物, 底物的普适性较好.

关键词 钯催化; 氧化偶氮苯; 酰基化; 绿色化学
\end{abstract}

\section{Palladium-Catalyzed Regioselective ortho-Acylation of Azoxybenzenes under Aqueous Conditions}

\author{
Chen, Xiaopei*, $\quad$ Ma, Zhiwei ${ }^{\dagger} \quad$ Wang, Chuanchuan $\quad$ Liu, Juntao $\quad$ Wu, Jinsong \\ (Faculty of Science, Henan University of Animal Husbandry and Economy, Zhengzhou 450046)
}

\begin{abstract}
A facile and efficient protocol for palladium-catalyzed ortho-acylation of azoxybenzenes has been developed under aqueous conditions. In this process, the alcohols were oxidized into the corresponding aldehydes in situ, which coupled with azoxybenzenes with excellent regioselectivity, affording the acylated azoxybenzenes in moderate to good yields. A variety of functional groups were tolerated in this procedure.

Keywords palladium catalysis; azoxybenzene; acylation; green chemistry
\end{abstract}

\section{Introduction}

Aryl ketones are important structural motifs in dyes, fragrances, fine chemicals, pharmaceuticals and agrochemicals. ${ }^{[1]}$ Conventionally, building such structure involves Friedel-Crafts acylation and oxidation of secondary alcohols by various oxidants. ${ }^{[2]}$ Although it is effective as documented in almost all organic chemistry textbooks, these processes suffer from some drawbacks, such as the formation of waste materials due to the use of excessive Lewis acids and oxidants (Scheme 1). The coupling of arenes with aliphatic olefins and carbon monoxide using ruthenium as a catalyst has provided an alternative pathway. ${ }^{[3]}$ The initial example developed by Moore and co-workers ${ }^{[4]}$ involves $\mathrm{Ru}_{3}(\mathrm{CO})_{12}$-catalyzed acylation of pyridine with $\mathrm{CO}$ and olefins. Recently, palladium-catalyzed acylation of $\mathrm{C}-\mathrm{H}$ bonds has been one of the most practical, atom- and step-economical methods in synthetic chemistry. In 2009, Cheng et al. ${ }^{[5]}$ first described palladium-catalyzed ortho-acylation reaction of arenes containing a pyridyl as a directing group with aryl aldehydes. Later, palladium-catalyzed $\mathrm{C}\left(\mathrm{sp}^{2}\right)-\mathrm{H}$ bond acylation of 2-arylpyridines, ${ }^{[5,6]}$ oximes, ${ }^{[7]}$ amides, ${ }^{[8]}$ acetanilides, ${ }^{[9]}$ indolizines, ${ }^{[10]}$ azobenzenes ${ }^{[11]}$ and benzothiazoles $^{[12]}$ using aldehydes, alcohols, $\alpha$-oxocarboxylic acids, carboxylic acids, $\alpha$-diketones, toluene derivatives and amines as acylation sources has been subsequently developed.

Azoxy compounds are important materials and useful intermediates in electronic devices due to their liquid crystalline properties. They also have wide applications in the fields of pharmaceuticals, analytic chemistry, functional materials, organic synthesis, and the dye industry. ${ }^{[13]}$ In view of their importance, a number of methods have been established to build a structural skeleton. ${ }^{[14]}$ Recently, Wang, Sun and Cui group ${ }^{[15]}$ realized ortho-acylation of azoxybenzenes using aldehydes, $\alpha$-oxocarboxylic acids and alcohols (Scheme 1). However, most of these processes are performed in organic solvents. From the perspective of green chemistry, water is an ideal chemical solvent, be-

\footnotetext{
* Corresponding author. E-mail: chenxp@hnuahe.edu.cn

Received May 9, 2019; revised June 14, 2019; published online July 9, 2019.

Project supported by the Key Scientific Research Project for Colleges and Universities of Henan Province (No. 20A150019) and the Doctoral Research Startup Fund of Henan University of Animal Husbandry and Economy (No. 53000168).

河南省高等学校重点科研项目计划(No. 20A150019)和河南牧业经济学院博士科研启动资金(No. 53000168)资助项目.

共同第一作者(These authors contributed equally to this work).
} 
cause it has natural, cheap, non-toxic, environmental characteristics. ${ }^{[16]}$ In the past few years, acylation reactions in water have occasionally been reported. ${ }^{[17]}$ Herein, we independently report a facile and efficient protocol to acylated azoxybenzenes via palladium-catalyzed $\mathrm{C}-\mathrm{H}$ bond activation using alcohols as acylation reagents under aqueous conditions (Scheme 1).

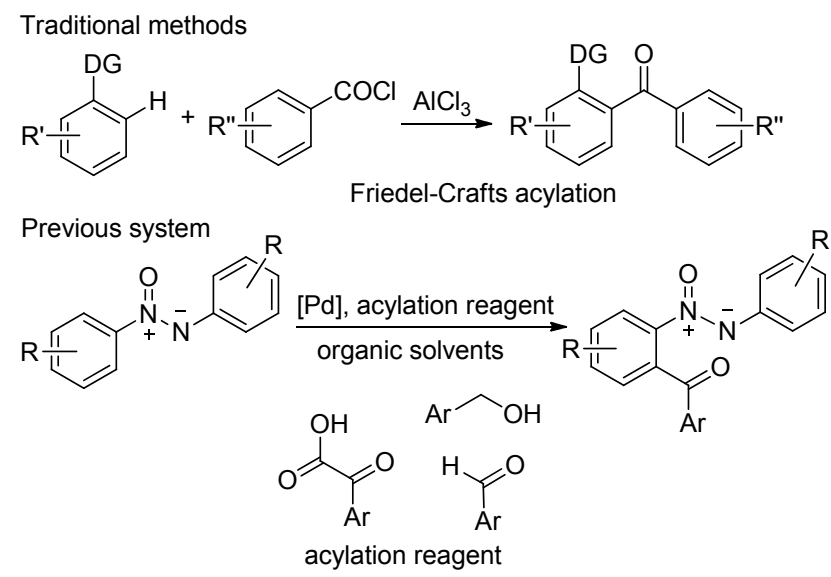

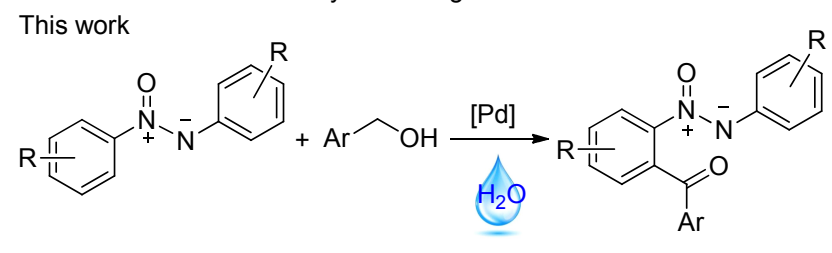

Scheme 1 Preparation of aryl ketones

\section{Results and discussion}

Our initial investigation was focused on the acylation of azoxybenzene (1a) with benzylalcohol (2a). The results were summarized in Table 1 . The desired product 3a was obtained in an isolated yield of $15 \%$ in water using 10 mol\% $\mathrm{PdCl}_{2}$ as catalyst and $70 \%$ tert-butyl hydroperoxide (TBHP) in water as oxidant (Entry 1, Table 1). To improve the solubility of the reactants in water, $5 \mathrm{~mol} \%$ sodium dodecyl sulfate (SDS) was added to the system. $\mathrm{Pd}(\mathrm{OAc})_{2}$ provided a higher yield compared to $\mathrm{PdCl}_{2}$ and $\mathrm{Pd}(\mathrm{TFA})_{2}$ (Entry 4 vs. Entries 2, 3, Table 1). Subsequently, various oxidants were tested in the presence of $\mathrm{Pd}(\mathrm{OAc})_{2}$ under air atmosphere (Entries 5 11). The yields could not be improved by using di-tert-butylperoxide (DTBP) and AgOAc (Entries 5,6). Only a trace amount of the desired product 3a were observed when $\mathrm{K}_{2} \mathrm{~S}_{2} \mathrm{O}_{8}$ and $\left(\mathrm{NH}_{4}\right)_{2} \mathrm{~S}_{2} \mathrm{O}_{8}$ as the oxidants (Entries 7, 8). benzoquinone (BQ), 2,3-dichloro5,6-dicyano-1,4-benzoquinone (DDQ) and $\mathrm{H}_{2} \mathrm{O}_{2}$ were found to be ineffective for this process (Entries 9 11). Other phase-transfer catalysts (PTC) showed that SDS is superior to other phase-transfer catalysts such as tetrabutylammonium bromide (TBAB), 18-crown-6, or tween 80 (Entries 12 14). Meanwhile, decreasing the temperature resulted in a slightly decrease in the yield (Entry 15). When the reaction was carried out under nitrogen atmosphere, the desired product 3a was also obtained in $75 \%$ yield (Entry 16), which indicated that oxygen molecule was tolerated. After surveying a variety of catalysts, oxidants, PTC and reaction temperatures, the optimized reaction conditions were identified as follows: $10 \mathrm{~mol} \%$ $\mathrm{Pd}(\mathrm{OAc})_{2}$ as catalyst, TBHP as oxidant, SDS as the phasetransfer catalyst, and $\mathrm{H}_{2} \mathrm{O}$ as solvent, at $100{ }^{\circ} \mathrm{C}$ under air atmosphere for $24 \mathrm{~h}$.

Table 1 Optimization of the reaction conditions ${ }^{a}$

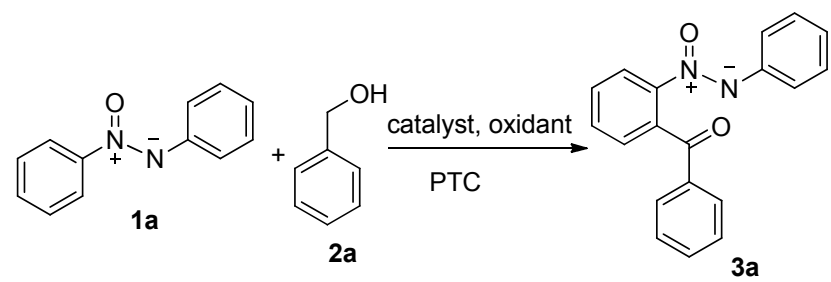

\begin{tabular}{lllll}
\hline Entry $^{a}$ & Catalyst & Oxidant & PTC & Yield $^{b} \%$ \\
\hline 1 & $\mathrm{PdCl}_{2}$ & TBHP & & 15 \\
2 & $\mathrm{PdCl}_{2}$ & TBHP & SDS & 35 \\
3 & $\mathrm{Pd}(\mathrm{TFA})_{2}$ & TBHP & SDS & 68 \\
4 & $\mathrm{Pd}(\mathrm{OAc})_{2}$ & TBHP & SDS & 80 \\
5 & $\mathrm{Pd}(\mathrm{OAc})_{2}$ & DBHP & SDS & 20 \\
6 & $\mathrm{Pd}(\mathrm{OAc})_{2}$ & $\mathrm{AgOAc}$ & $\mathrm{SDS}$ & 15 \\
7 & $\mathrm{Pd}(\mathrm{OAc})_{2}$ & $\mathrm{~K}_{2} \mathrm{~S}_{2} \mathrm{O}_{8}$ & $\mathrm{SDS}$ & Trace \\
8 & $\mathrm{Pd}(\mathrm{OAc})_{2}$ & $\left(\mathrm{NH}_{4}\right)_{2} \mathrm{~S}_{2} \mathrm{O}_{8}$ & $\mathrm{SDS}$ & Trace \\
9 & $\mathrm{Pd}(\mathrm{OAc})_{2}$ & $\mathrm{BQ}$ & $\mathrm{SDS}$ & $\mathrm{ND}^{e}$ \\
10 & $\mathrm{Pd}(\mathrm{OAc})_{2}$ & $\mathrm{DDQ}$ & $\mathrm{SDS}$ & $\mathrm{ND}^{e}$ \\
11 & $\mathrm{Pd}(\mathrm{OAc})_{2}$ & $\mathrm{H}_{2} \mathrm{O}_{2}$ & SDS & $\mathrm{ND}^{e}$ \\
12 & $\mathrm{Pd}(\mathrm{OAc})_{2}$ & $\mathrm{TBHP}$ & $\mathrm{TBAB}$ & 20 \\
13 & $\mathrm{Pd}(\mathrm{OAc})_{2}$ & TBHP & $18-C r o w n-6$ & 42 \\
14 & $\mathrm{Pd}(\mathrm{OAc})_{2}$ & TBHP & Tween 80 & 10 \\
$15^{c}$ & $\mathrm{Pd}(\mathrm{OAc})_{2}$ & TBHP & SDS & 65 \\
$16^{d}$ & $\mathrm{Pd}(\mathrm{OAc})_{2}$ & TBHP & SDS & 75 \\
\hline
\end{tabular}

${ }^{a}$ Reaction conditions: 1a $(0.2 \mathrm{mmol}), 2 \mathrm{a}(0.6 \mathrm{mmol})$, catalyst $(10 \mathrm{~mol} \%)$, oxidant (4.5 equiv.), PTC ( $5 \mathrm{~mol} \%)$ and water $(2.0 \mathrm{~mL})$, under air, $100{ }^{\circ} \mathrm{C}, 24$ h. ${ }^{b}$ Isolated yield. ${ }^{c} 80{ }^{\circ} \mathrm{C} .{ }^{d}$ Nitrogen protection. ${ }^{e} \mathrm{ND}$ is not detected.

With the optimal reaction conditions in hand, the generality and the scope of the substrates for this transformation were tested(Table 2, $\mathbf{3 a} \sim \mathbf{3 m}$ ). A wide range of primary alcohols were firstly evaluated in reaction with azoxybenzene (1a). In general, the reactions of primary alcohols with electron-donating groups (such as $\mathrm{CH}_{3}, \mathrm{OCH}_{3}$ ) or weak electron-withdrawing groups (such as $\mathrm{F}, \mathrm{Cl}, \mathrm{Br}$ ) on the aromatic ring afforded the desired products in $55 \%$ $78 \%$ yields $(\mathbf{3 b} \sim \mathbf{3 f})$. The presence of the strong electronwithdrawing group, such as (4-nitrophenyl)methanol, restrained the reaction, providing the product in only $32 \%$ yield (3g). Steric hindrance on the aryl group of primary alcohols had no significant effect, furnishing the corresponding products in moderate yields $(\mathbf{3 h} \sim \mathbf{3 l})$. Notably, when azoxybenzene (1a) was coupled with (3-nitrophenyl)methanol (2l) under the optimized reaction conditions, a satisfactory yield of the corresponding product $\mathbf{3 I}$ was achieved. Disubstituted benzyl alcohols 3i provided the desired products in $63 \%$ yield. Aliphatic alcohol exhibited low efficiency in this transformation. For example, the $n$-butyl alcohol delivered $34 \%$ yield of $\mathbf{3 m}$. 
Table 2 Scope of azoxybenzenes and alcohols ${ }^{a}$
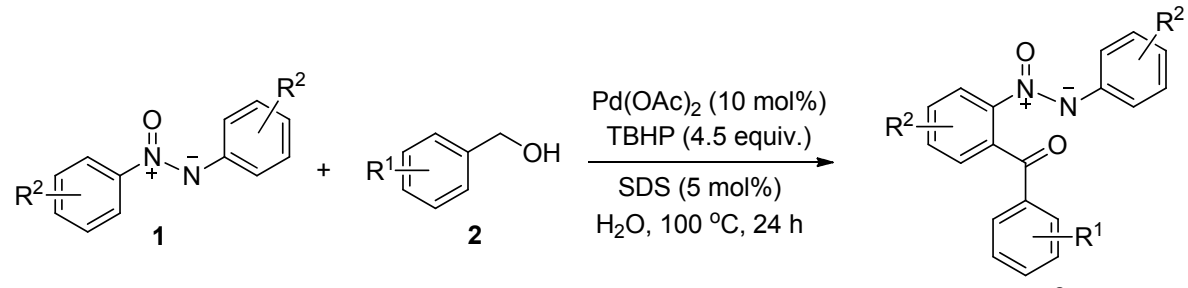

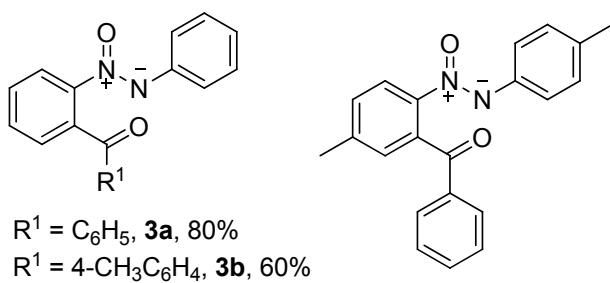

$\mathrm{R}^{1}=4-\mathrm{CH}_{3} \mathrm{OC}_{6} \mathrm{H}_{4}, 3 \mathrm{c}, 55 \%$

$\mathrm{R}^{1}=4-\mathrm{FC}_{6} \mathrm{H}_{4}, 3 \mathrm{~d}, 72 \%$

$\mathrm{R}^{1}=4-\mathrm{ClC}_{6} \mathrm{H}_{4}, \mathbf{3 e}, 78 \%$

$\mathrm{R}^{1}=4-\mathrm{BrC}_{6} \mathrm{H}_{4}, \mathbf{3 f}, 75 \%$

$\mathrm{R}^{1}=4-\mathrm{O}_{2} \mathrm{NC}_{6} \mathrm{H}_{4}, \mathbf{3 g}, 32 \%$

$\mathrm{R}^{1}=2-\mathrm{CH}_{3} \mathrm{C}_{6} \mathrm{H}_{4}, 3 \mathbf{h}, 58 \%$

$\mathrm{R}^{1}=2,4-\mathrm{Cl}_{2} \mathrm{C}_{6} \mathrm{H}_{3}, 3 \mathbf{i}, 63 \%$

$\mathrm{R}^{1}=3-\mathrm{CH}_{3} \mathrm{OC}_{6} \mathrm{H}_{4}, \mathbf{3 j}, 65 \%$

$\mathrm{R}^{1}=3-\mathrm{ClC}_{6} \mathrm{H}_{4}, \mathbf{3 k}, 59 \%$

$\mathrm{R}^{1}=3-\mathrm{O}_{2} \mathrm{NC}_{6} \mathrm{H}_{4}, 3 \mathrm{I}, 56 \%$

$\mathrm{R}^{1}=\mathrm{C}_{3} \mathrm{H}_{7}, 3 \mathrm{~m}, 34 \%$<smiles>CC(C)c1ccc(N[N+](=O)c2ccc(C(C)C)cc2C(=O)c2ccccc2)cc1</smiles>

3o $(68 \%)$

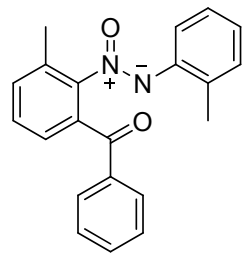

3r $(70 \%)$

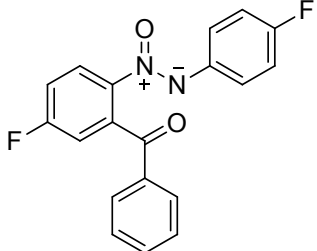

3 s $(32 \%)$<smiles></smiles><smiles></smiles>

3q $(73 \%)$

${ }^{a}$ Reaction conditions: $1(0.2 \mathrm{mmol}), 2(0.4 \mathrm{mmol}), \mathrm{Pd}(\mathrm{OAc})_{2}(10 \mathrm{~mol} \%)$, TBHP $(0.9 \mathrm{mmol}), \mathrm{SDS}(5 \mathrm{~mol} \%), \mathrm{H}_{2} \mathrm{O}(2.0 \mathrm{~mL})$, under air, $100{ }^{\circ} \mathrm{C}, 24 \mathrm{~h} .{ }^{b}$ Isolated yield.

Heteroarylaldehydes, such as 2-pyridinecarboxaldehyde, 2-furanaldehyde and 2-thiophenecarboxaldehyde, were not suitable substrates. To expand the scope of this method, some typical disubstituted azoxybenzenes were examined. The results demonstrated that azoxy derivatives with electron-donating groups gave better yields than those with electron-withdrawing groups. For instance, the reactions of 4,4'-dimethylazoxybenzene, 4,4'-diisopropylazoxybenzene 4,4'-dimethoxyazoxybenzene, 3,3'-dimethylazoxybenzene, and 2,2'-dimethylazoxybenzene provided the corresponding products in $68 \% \sim 73 \%$ yields $(3 \mathbf{n} \sim 3 \mathbf{r})$. The electron-withdrawing group in the substrate, such as $\mathrm{F}$ and $\mathrm{Br}$, provided $3 \mathbf{s}$ and $3 \mathbf{t}$ in $32 \%$ and $33 \%$ yields.

To clarify the reaction mechanism, some control experiments were carried out (Scheme 2). When the palladium-catalyzed acylation of 1a with 2a was carried out in the absence of TBHP (Scheme 2a, Condition A), no acylated product 3a was detected. Moreover, addition of a radical scavenger 2,2,6,6-tetramethylpiperidyl-1-oxyl (TEMPO) to the reaction mixture under the standard reaction conditions made this acylation suppressed, suggesting the possibility of a radical process (Scheme 2a, Condition B). Furthermore, it was found that benzaldehyde was formed under the reaction conditions without the azoxybenzene (Scheme $2 b$ ). Then, the reaction between azoxybenzene (1a) and benzaldehyde were examined, and the product $\mathbf{3 a}$ was provided in $75 \%$ yield (Scheme $2 \mathrm{c}$ ).

Based on the results obtained and the literature, ${ }^{[15,17,18]} \mathrm{a}$ tentative reaction mechanism for the palladium-catalyzed ortho- $\mathrm{C}-\mathrm{H}$ acylation of azoxybenzene (1a) was proposed

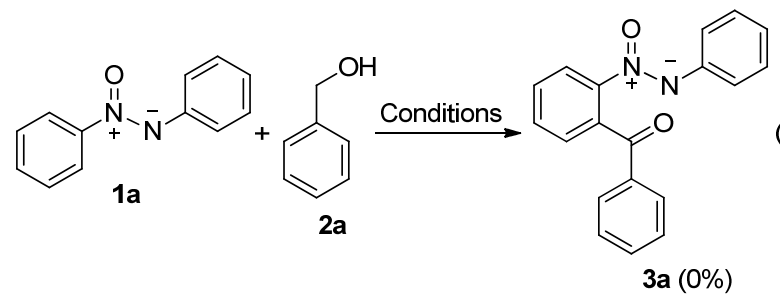

Condition $\mathrm{A}: \mathrm{Pd}(\mathrm{OAc})_{2}(10 \mathrm{~mol} \%)$, SDS (5 mol\%), $\mathrm{H}_{2} \mathrm{O}$, $100^{\circ} \mathrm{C}, 24 \mathrm{~h}$

Condition $\mathrm{B}: \mathrm{Pd}(\mathrm{OAc})_{2}$ (10 mol\%), TBHP (4.5 equiv.),

SDS ( 5 mol\%), TEMPO ( 4.0 equiv.), $\mathrm{H}_{2} \mathrm{O}, 100^{\circ} \mathrm{C}, 24 \mathrm{~h}$

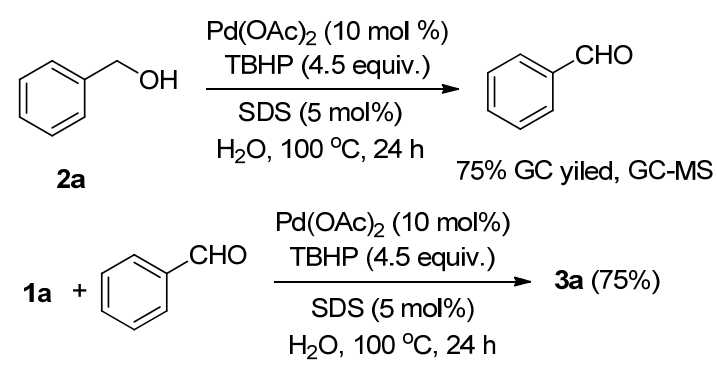

Scheme 2 Control experiments

and depicted in Scheme 3. The palladium catalyst reacted with azoxybenzene by chelation-directed $\mathrm{C}-\mathrm{H}$ activation to generate complex A (Figure 1). Meanwhile, with the effect of TBHP, the benzaldehyde, which was produced from the oxidation of benzylalcohol (2a), generated an acyl radical. Then, the complex $\mathbf{A}$ would react with the acyl radical, affording the oxidative addition product as the di- 
meric $\mathrm{Pd}^{\mathrm{III}}$ or reactive $\mathrm{Pd}^{\mathrm{IV}}$ species $\mathbf{B}^{\left[{ }^{[1]}\right.}$ Finally, the reductive elimination of intermediate $\mathbf{B}$ afforded coupling product 3a and regenerated Pd(II) for next cycle.

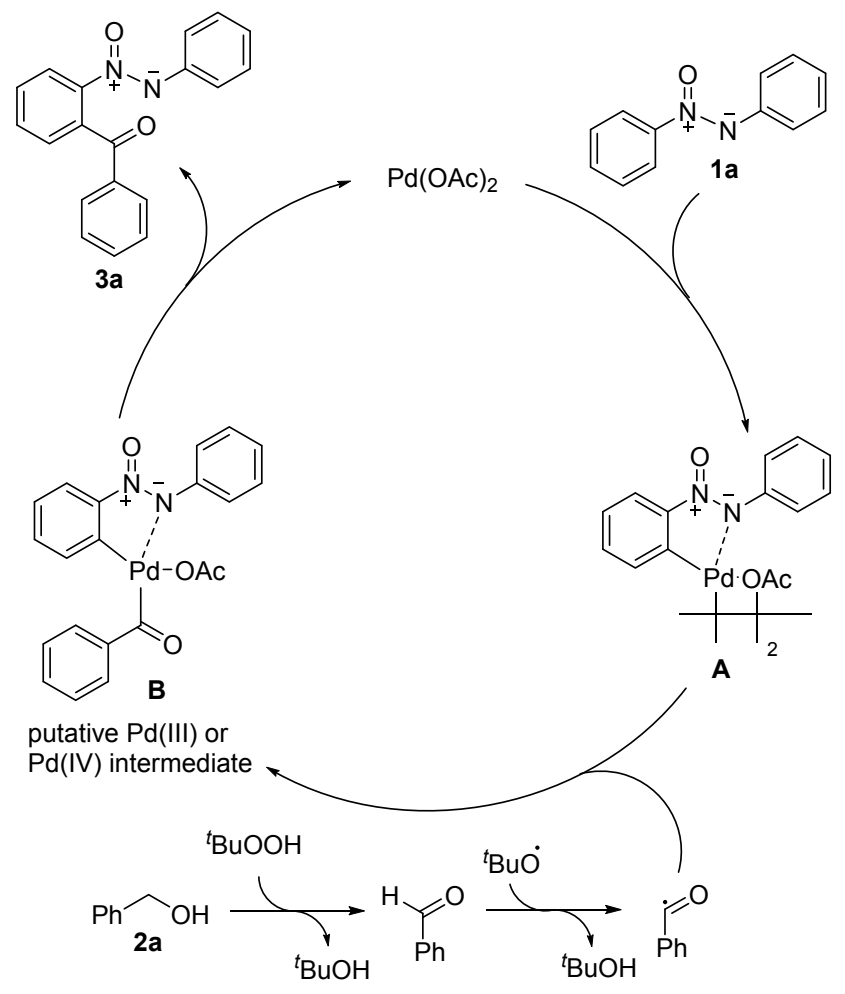

Scheme 3 Proposed reaction mechanism

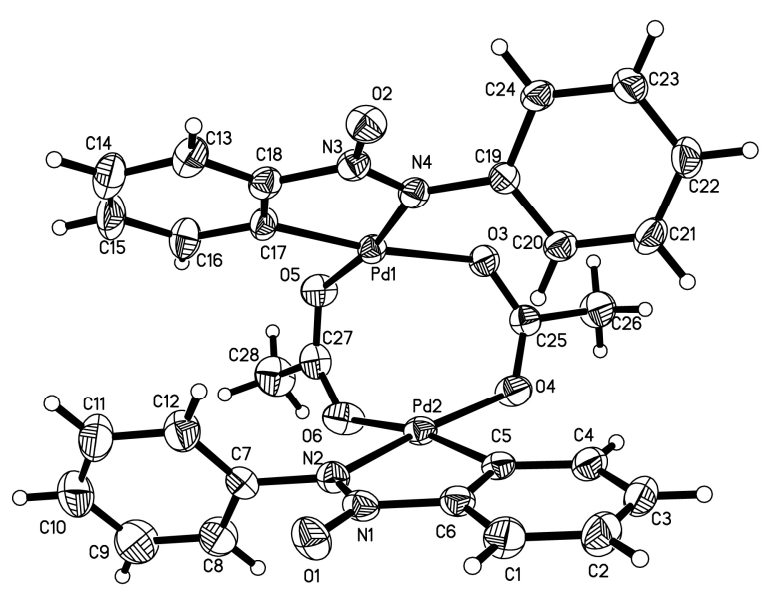

Figure 1 X-Ray molecular structures of complex $\mathbf{A}$

\section{Conclusions}

In summary, a simple, efficient and environmentally benign protocol for the synthesis of acylated azoxybenzenes via Pd-catalyzed $\mathrm{C}-\mathrm{H}$ regioselective functionalization has been developed. The method is characterized by its wide substrate scope, high regioselectivity, mild reaction conditions, and readily available starting materials. Further investigations to expand the substrate scope and application of such chemistry in organic synthesis are underway.

\section{Experimental section}

\subsection{General information}

Melting points were measured on a microscopic apparatus and were uncorrected. ${ }^{1} \mathrm{H}$ NMR spectra were recorded on a $400 \mathrm{MHz}$ spectrometer in deuterated chloroform. The chemical shifts $(\delta)$ are reported relative to tetramethylsilane. ${ }^{13} \mathrm{C}$ NMR spectra were recorded using a 100 $\mathrm{MHz}$ spectrometer. The chemical shifts are reported relative to residual $\mathrm{CHCl}_{3} \quad\left(\delta_{\mathrm{C}}=77.00\right)$. High-resolution mass spectrometry (HRMS) was performed on a Q-TOF spectrometer with micromass MS software using electrospray ionization (ESI). X-ray analysis was performed with a single-crystal X-ray diffractometer. Unless otherwise noted, all of the reagents were purchased from commercial suppliers and used without purification.

\subsection{General procedure for preparation of azoxy- benzenes}

All of the azoxybenzenes were prepared from arylamines, according to the literature. ${ }^{[20]} \mathrm{H}_{2} \mathrm{O}_{2}(30 \%, 0.92 \mathrm{~mL}$, $9.00 \mathrm{mmol})$ was added to a solution of arylamine $(0.27$ $\mathrm{mL}, 3.00 \mathrm{mmol})$ and $\mathrm{SeO}_{2}(33.3 \mathrm{mg}, 0.30 \mathrm{mmol})$ in $\mathrm{MeOH}$ $(10 \mathrm{~mL})$. The reaction mixture was stirred at room temperature (r.t.) until complete consumption of the starting material, which was followed by thin-layer chromatography (TLC) $(20 \mathrm{~h})$. The solvent was evaporated under vacuum. The residue was partitioned between $\mathrm{CH}_{2} \mathrm{Cl}_{2}(20 \mathrm{~mL})$ and $\mathrm{H}_{2} \mathrm{O}(20 \mathrm{~mL})$. The organic layer was separated, and the aqueous phase was extracted with $\mathrm{CH}_{2} \mathrm{Cl}_{2}(20 \mathrm{~mL} \times 2)$. The combined organic phase were dried $\left(\mathrm{MgSO}_{4}\right)$ and concentrated in vacuo. The crude product was purified by column chromatography on silica gel $\left(\mathrm{CH}_{2} \mathrm{Cl}_{2}\right.$-pentane, $V: V=1: 10)$ to afford the desired azoxybenzene derivatives.

4.3 Typical procedure for the acylation of azobenzenes

Azoxybenzene (1a, $39.6 \mathrm{mg}, 0.20 \mathrm{mmol}$ ), benzyl alcohol (2a, $63 \mu \mathrm{L}, 0.40 \mathrm{mmol}), \mathrm{Pd}(\mathrm{OAc})_{2}(4.48 \mathrm{mg}, 0.02$ $\mathrm{mmol})$, TBHP (126 uL, 70\%, $0.9 \mathrm{mmol})$, SDS (2.88 mg, $0.01 \mathrm{mmol})$ and $\mathrm{H}_{2} \mathrm{O}(2.0 \mathrm{~mL})$ were mixed in a dry reaction tube. The mixture was stirred at $100{ }^{\circ} \mathrm{C}$ under air for $24 \mathrm{~h}$. When the reaction was completed, the crude mixture was cooled to room temperature. The mixture was extracted with ethyl acetate three times. The filtrate was concentrated in vacuum and the resulting residue was purified by preparative thin layer chromatography (silica gel, ethyl acetate/petroleum ether, $V: V=1: 10)$ to give the desired product 1-(2-benzoylphenyl)-2-phenyldiazene 1-oxide $(3 \mathbf{a})^{[15]}$ as a yellow viscous liquid $(48.3 \mathrm{mg}, 80 \%) .{ }^{1} \mathrm{H}$ NMR $\left(400 \mathrm{MHz}, \mathrm{CDCl}_{3}\right) \delta: 8.24 \sim 8.22(\mathrm{~m}, 1 \mathrm{H}), 7.76(\mathrm{~d}$, $J=7.4 \mathrm{~Hz}, 2 \mathrm{H}), 7.70 \sim 7.64(\mathrm{~m}, 4 \mathrm{H}), 7.53 \sim 7.51(\mathrm{~m}, 1 \mathrm{H})$, $7.46(\mathrm{t}, J=6.2 \mathrm{~Hz}, 1 \mathrm{H}), 7.36(\mathrm{t}, J=6.1 \mathrm{~Hz}, 2 \mathrm{H}), 7.29(\mathrm{~d}$, $J=3.2 \mathrm{~Hz}, 2 \mathrm{H}) ;{ }^{13} \mathrm{C}$ NMR $\left(100 \mathrm{MHz}, \mathrm{CDCl}_{3}\right) \delta: 194.0$, $147.2,143.1,136.9,134.8,133.1,131.4,130.5,129.9$, $128.9,128.8,128.5,125.0,123.4$; HRMS (ESI) calcd for $\mathrm{C}_{19} \mathrm{H}_{15} \mathrm{~N}_{2} \mathrm{O}_{2}[\mathrm{M}+\mathrm{H}]^{+}$303.1128, found 303.1133 . 
1 -(2-(4-Methylbenzoyl)phenyl)-2-phenyldiazene 1-oxide (3b): ${ }^{[15]}$ yellow liquid $(37.9 \mathrm{mg}, 60 \%) .{ }^{1} \mathrm{H}$ NMR $\left(400 \mathrm{MHz}, \mathrm{CDCl}_{3}\right) \delta: 8.22 \sim 8.21(\mathrm{~m}, 1 \mathrm{H}), 7.73 \sim 7.71(\mathrm{~m}$, $2 \mathrm{H}), 7.65 \sim 7.63(\mathrm{~m}, 4 \mathrm{H}), 7.51 \sim 7.48(\mathrm{~m}, 1 \mathrm{H}), 7.32 \sim 7.27$ $(\mathrm{m}, 3 \mathrm{H}), 7.167 .16(\mathrm{~d}, J=7.9 \mathrm{~Hz}, 2 \mathrm{H}), 2.33(\mathrm{~s}, 3 \mathrm{H}) ;{ }^{13} \mathrm{C}$ NMR $\left(100 \mathrm{MHz}, \mathrm{CDCl}_{3}\right) \delta: 194.1,144.3,143.6,135.4$, $134.8,131.6,130.7,130.2,129.6,129.4,128.8,125.4$, 123.7, 22.0; HRMS (ESI) calcd for $\mathrm{C}_{20} \mathrm{H}_{17} \mathrm{~N}_{2} \mathrm{O}_{2}[\mathrm{M}+\mathrm{H}]^{+}$ 317.1285 , found 317.1289 .

1-(2-(4-Methoxybenzoyl)phenyl)-2-phenyldiazene 1-oxide (3c): ${ }^{[15]}$ yellow liquid $(36.5 \mathrm{mg}, 55 \%)$. ${ }^{1} \mathrm{H}$ NMR $\left(400 \mathrm{MHz}, \mathrm{CDCl}_{3}\right) \delta: 8.22 \sim 8.20(\mathrm{~m}, 1 \mathrm{H}), 7.75(\mathrm{~d}, J=8.4$ $\mathrm{Hz}, 3 \mathrm{H}), 7.63 \sim 7.61(\mathrm{~m}, 2 \mathrm{H}), 7.49 \sim 7.47(\mathrm{~m}, 1 \mathrm{H}), 7.31 \sim$ $7.27(\mathrm{~m}, 3 \mathrm{H}), 6.84(\mathrm{~d}, J=8.8 \mathrm{~Hz}, 2 \mathrm{H}), 3.79(\mathrm{~s}, 3 \mathrm{H}) ;{ }^{13} \mathrm{C}$ NMR $\left(100 \mathrm{MHz}, \mathrm{CDCl}_{3}\right) \delta: 193.2,163.9,143.6,135.5$, $131.6,131.6,130.6,130.4,130.2,129.1,128.9,125.4$, 123.7, 114.20, 55.8; HRMS (ESI) calcd for $\mathrm{C}_{20} \mathrm{H}_{17} \mathrm{~N}_{2} \mathrm{O}_{3}$ $[\mathrm{M}+\mathrm{H}]^{+}$333.1234, found 333.1238.

1-(2-(4-Fluorobenzoyl)phenyl)-2-phenyldiazene 1-oxide (3d): ${ }^{[15]}$ yellow liquid $(46.1 \mathrm{mg}, 72 \%) .{ }^{1} \mathrm{H}$ NMR (400 $\left.\mathrm{MHz}, \mathrm{CDCl}_{3}\right) \delta: 8.24 \sim 8.22(\mathrm{~m}, 1 \mathrm{H}), 7.77 \sim 7.73(\mathrm{~m}, 4 \mathrm{H})$, $7.66(\mathrm{t}, J=9.3 \mathrm{~Hz}, 2 \mathrm{H}), 7.50 \sim 7.48(\mathrm{~m}, 1 \mathrm{H}), 7.31 \sim 7.29$ $(\mathrm{m}, 3 \mathrm{H}), 7.03(\mathrm{t}, J=10.3 \mathrm{~Hz}, 2 \mathrm{H}) ;{ }^{13} \mathrm{C}$ NMR $(100 \mathrm{MHz}$, $\left.\mathrm{CDCl}_{3}\right) \delta: 192.9,167.3,164.7,143.5,134.8,133.8,131.8$, $131.8,131.7,130.5,129.1,128.9,125.4,123.8,116.2$, 115.9; HRMS (ESI) calcd for $\mathrm{C}_{19} \mathrm{H}_{14} \mathrm{FN}_{2} \mathrm{O}_{2}[\mathrm{M}+\mathrm{H}]^{+}$ 321.1034 , found 321.1037 .

1-(2-(4-Chlorobenzoyl)phenyl)-2-phenyldiazene 1-oxide (3e) $:^{[15]}$ yellow liquid $(52.4 \mathrm{mg}, 78 \%)$, m.p. 64.6 $65.2{ }^{\circ} \mathrm{C} .{ }^{1} \mathrm{H}$ NMR $\left(400 \mathrm{MHz}, \mathrm{CDCl}_{3}\right) \delta: 8.26 \sim 8.23(\mathrm{~m}$, 1H), 7.75 (d, $J=8.0 \mathrm{~Hz}, 2 \mathrm{H}), 7.70(\mathrm{~d}, J=8.4 \mathrm{~Hz}, 2 \mathrm{H}), 7.65$ $(\mathrm{t}, J=6.6 \mathrm{~Hz}, 3 \mathrm{H}), 7.51 \sim 7.49(\mathrm{~m}, 1 \mathrm{H}), 7.34 \sim 7.31(\mathrm{~m}$, $5 \mathrm{H}) ;{ }^{13} \mathrm{C}$ NMR $\left(100 \mathrm{MHz}, \mathrm{CDCl}_{3}\right) \delta: 193.1,143.4,139.8$, $135.7,134.6,131.8,131.1,130.5,130.5,129.2,129.1$, 129.0, 125.4, 123.8; HRMS (ESI) calcd for $\mathrm{C}_{19} \mathrm{H}_{14} \mathrm{ClN}_{2} \mathrm{O}_{2}$ $[\mathrm{M}+\mathrm{H}]^{+}, 337.0738$, found 337.0743 .

1-(2-(4-Bromobenzoyl)phenyl)-2-phenyldiazene 1-oxide (3f): $:{ }^{[15]}$ yellow liquid $(57.0 \mathrm{mg}, 75 \%)$. m.p. $62.5 \sim 63.4{ }^{\circ} \mathrm{C}$; ${ }^{1} \mathrm{H}$ NMR $\left(400 \mathrm{MHz}, \mathrm{CDCl}_{3}\right) \delta: 8.25 \sim 8.23(\mathrm{~m}, 1 \mathrm{H}), 7.74$ (d, $J=7.4 \mathrm{~Hz}, 2 \mathrm{H}), 7.66$ (t, $J=4.7 \mathrm{~Hz}, 2 \mathrm{H}), 7.61(\mathrm{~d}, J=8.3$ $\mathrm{Hz}, 2 \mathrm{H}), 7.49(\mathrm{~d}, J=8.0 \mathrm{~Hz}, 3 \mathrm{H}), 7.35 \sim 7.30(\mathrm{~m}, 3 \mathrm{H}) .{ }^{13} \mathrm{C}$ NMR $\left(100 \mathrm{MHz}, \mathrm{CDCl}_{3}\right) \delta: 193.3,143.4,136.2,134.6$, $132.2,131.8,131.1,130.6,130.5,129.1,129.0,128.6$, 125.4, 123.8; HRMS (ESI) calcd for $\mathrm{C}_{19} \mathrm{H}_{14} \mathrm{BrN}_{2} \mathrm{O}_{2}[\mathrm{M}+$ $\mathrm{H}]^{+}$381.0233, found 381.0236.

1-(2-(4-Nitrobenzoyl)phenyl)-2-phenyldiazene 1-oxide $(\mathbf{3 g})::^{[15]}$ yellow liquid $(22.2 \mathrm{mg}, 32 \%) .{ }^{1} \mathrm{H}$ NMR (400 $\left.\mathrm{MHz}, \mathrm{CDCl}_{3}\right) \delta: 8.30 \sim 8.28(\mathrm{~m}, 4 \mathrm{H}), 8.18(\mathrm{~d}, J=8.6 \mathrm{~Hz}$, $2 \mathrm{H}), 7.89(\mathrm{~d}, J=8.6 \mathrm{~Hz}, 2 \mathrm{H}), 7.77 \sim 7.70(\mathrm{~m}, 4 \mathrm{H}), 7.56 \sim$ $7.54(\mathrm{~m}, 1 \mathrm{H}), 7.33 \sim 7.31(\mathrm{~m}, 3 \mathrm{H}) ;{ }^{13} \mathrm{C}$ NMR $(100 \mathrm{MHz}$, $\left.\mathrm{CDCl}_{3}\right) \delta: 190.4,150.4,143.2,142.0,133.9,132.1,131.6$, 130.9, 129.7, 129.2, 125.4, 124.1, 123.8; HRMS (ESI) calcd for $\mathrm{C}_{19} \mathrm{H}_{14} \mathrm{~N}_{3} \mathrm{O}_{4}[\mathrm{M}+\mathrm{H}]^{+}$348.0979, found 348.0981 .

1-(2-(2-Methylbenzoyl)phenyl)-2-phenyldiazene 1 oxide (3h): ${ }^{[15]}$ yellow liquid $(36.7 \mathrm{mg}, 58 \%)$. ${ }^{1} \mathrm{H}$ NMR (400 $\left.\mathrm{MHz} \mathrm{CDCl}_{3}\right) \delta: 8.05 \sim 8.03(\mathrm{~m}, 1 \mathrm{H}), 7.64 \sim 7.60(\mathrm{~m}, 3 \mathrm{H})$,
$7.57 \sim 7.54(\mathrm{~m}, 2 \mathrm{H}), 7.32 \sim 7.25(\mathrm{~m}, 5 \mathrm{H}), 7.14 \sim 7.07(\mathrm{~m}$, 2H), $2.53(\mathrm{~s}, 3 \mathrm{H}) ;{ }^{13} \mathrm{C} \mathrm{NMR}\left(100 \mathrm{MHz}, \mathrm{CDCl}_{3}\right) \delta: 195.7$, $148.3,143.7,140.2,136.6,136.1,132.2,131.1,131.4$, $131.3,130.7,130.0,128.7,125.7,123.8,21.5$; HRMS (ESI) calcd for $\mathrm{C}_{20} \mathrm{H}_{17} \mathrm{~N}_{2} \mathrm{O}_{2}[\mathrm{M}+\mathrm{H}]^{+}$317.1285; found 317.1289 .

1-(2-(2,4-Dichlorobenzoyl)phenyl)-2-phenyldiazene 1oxide (3i): yellow liquid (46.6 mg, 63\%). ${ }^{1} \mathrm{H}$ NMR (400 $\left.\mathrm{MHz}, \mathrm{CDCl}_{3}\right) \delta: 8.06(\mathrm{~d}, J=7.4 \mathrm{~Hz}, 1 \mathrm{H}), 7.79 \sim 7.77(\mathrm{~m}$, 2H), $7.68 \sim 7.63(\mathrm{~m}, 3 \mathrm{H}), 7.48(\mathrm{~d}, J=8.3 \mathrm{~Hz}, 1 \mathrm{H}), 7.37 \sim$ $7.31(\mathrm{~m}, 4 \mathrm{H}), 7.16 \sim 7.14(\mathrm{~m}, 1 \mathrm{H}) ;{ }^{13} \mathrm{C} \mathrm{NMR}(100 \mathrm{MHz}$, $\left.\mathrm{CDCl}_{3}\right) \delta: 191.4,143.6,138.6,134.8,134.7,134.6,132.5$, $131.9,131.3,130.6,129.9,128.9,127.2,125.5,123.9$; HRMS (ESI) calcd for $\mathrm{C}_{19} \mathrm{H}_{13} \mathrm{Cl}_{2} \mathrm{~N}_{2} \mathrm{O}_{2} \quad[\mathrm{M}+\mathrm{H}]$ 371.0349 , found 371.0352 .

1-(2-(3-Methoxybenzoyl)phenyl)-2-phenyldiazene 1oxide (3j): yellow liquid (43.1 mg, 65\%). ${ }^{1} \mathrm{H}$ NMR (400 $\left.\mathrm{MHz}, \mathrm{CDCl}_{3}\right) \delta: 8.24 \sim 8.20(\mathrm{~m}, 1 \mathrm{H}), 7.73 \sim 7.71(\mathrm{~m}, 2 \mathrm{H})$, $7.66 \sim 7.63(\mathrm{~m}, 2 \mathrm{H}), 7.53 \sim 7.51(\mathrm{~m}, 1 \mathrm{H}), 7.38(\mathrm{~s}, 1 \mathrm{H})$, $7.33 \sim 7.31(\mathrm{~m}, 3 \mathrm{H}), 7.25 \sim 7.20(\mathrm{~m}, 2 \mathrm{H}), 7.04 \sim 6.99(\mathrm{~m}$, 1H), $3.75(\mathrm{~s}, 3 \mathrm{H}) ;{ }^{13} \mathrm{C}$ NMR $\left(100 \mathrm{MHz}, \mathrm{CDCl}_{3}\right) \delta: 194.1$, $160.1,143.5,138.7,135.1,131.7,130.9,129.8,129.2$, 128.8, 125.3, 123.7, 122.1, 120.2, 112.9, 55.8; HRMS (ESI) calcd for $\mathrm{C}_{20} \mathrm{H}_{17} \mathrm{~N}_{2} \mathrm{O}_{3}[\mathrm{M}+\mathrm{H}]^{+}$333.1234, found 333.1238 .

1-(2-(3-Chlorobenzoyl)phenyl)-2-phenyldiazene 1-oxide (3k): ${ }^{[15]}$ yellow liquid (39.6 mg, 59\%). ${ }^{1} \mathrm{H}$ NMR (400 $\left.\mathrm{MHz}, \mathrm{CDCl}_{3}\right) \delta: 8.26 \sim 8.24(\mathrm{~m}, 1 \mathrm{H}), 7.77 \sim 7.74(\mathrm{~m}, 3 \mathrm{H})$, $7.68 \sim 7.66(\mathrm{~m}, 2 \mathrm{H}), 7.58(\mathrm{~d}, J=7.8 \mathrm{~Hz}, 1 \mathrm{H}), 7.51 \sim 7.49$ $(\mathrm{m}, 1 \mathrm{H}), 7.42(\mathrm{~d}, J=7.9 \mathrm{~Hz}, 1 \mathrm{H}), 7.35 \sim 7.25(\mathrm{~m}, 4 \mathrm{H}) ;{ }^{13} \mathrm{C}$ NMR $\left(100 \mathrm{MHz}, \mathrm{CDCl}_{3}\right) \delta: 193.0,143.4,139.0,135.2$, $134.5,133.3,131.8,131.2,130.5,130.2,129.1,129.0$, 129.0, 127.2, 125.4, 123.8; HRMS (ESI) calcd for $\mathrm{C}_{19} \mathrm{H}_{14} \mathrm{ClN}_{2} \mathrm{O}_{2}[\mathrm{M}+\mathrm{H}]^{+}$337.0738, found 337.0743.

1-(2-(3-Nitrobenzoyl)phenyl)-2-phenyldiazene 1-oxide (31): yellow liquid (38.8 mg, 56\%). ${ }^{1} \mathrm{H}$ NMR (400 MHz, $\left.\mathrm{CDCl}_{3}\right) \delta: 8.57 \sim 8.56(\mathrm{~m}, 1 \mathrm{H}), 8.30 \sim 8.27(\mathrm{~m}, 2 \mathrm{H}), 8.03$ $(\mathrm{d}, J=7.7 \mathrm{~Hz}, 1 \mathrm{H}), 7.78 \sim 7.75(\mathrm{~m}, 2 \mathrm{H}), 7.73 \sim 7.69(\mathrm{~m}$, $2 \mathrm{H}), 7.57 \sim 7.52(\mathrm{~m}, 2 \mathrm{H}), 7.34 \sim 7.30(\mathrm{~m}, 3 \mathrm{H}) ;{ }^{13} \mathrm{C} \mathrm{NMR}$ $\left(100 \mathrm{MHz}, \mathrm{CDCl}_{3}\right) \delta: 192.0,148.7,147.5,143.3,138.9$, $134.3,133.7,132.1,131.6,130.8,130.1,129.1,129.0$, 127.5, 125.5, 124.0, 123.7; HRMS (ESI) calcd for $\mathrm{C}_{19} \mathrm{H}_{14} \mathrm{~N}_{3} \mathrm{O}_{4}[\mathrm{M}+\mathrm{H}]^{+}$348.0979, found 348.0982.

1-(2-Butyrylphenyl)-2-phenyldiazene 1-oxide (3m): $:^{[15]}$ yellow liquid (18.1 mg, 34\%). ${ }^{1} \mathrm{H}$ NMR (400 $\mathrm{MHz}, \mathrm{CDCl}_{3}$ ) $\delta: 8.10(\mathrm{~d}, J=8.1 \mathrm{~Hz}, 3 \mathrm{H}), 7.58 \sim 7.56(\mathrm{~m}, 2 \mathrm{H}), 7.49 \sim$ $7.46(\mathrm{~m}, 2 \mathrm{H}), 7.43 \sim 7.40(\mathrm{~m}, 2 \mathrm{H}), 2.73(\mathrm{t}, J=7.0 \mathrm{~Hz}, 2 \mathrm{H})$, 1.71 (q, $J=10.4,3.0 \mathrm{~Hz}, 2 \mathrm{H}), 0.92$ (t, $J=7.4 \mathrm{~Hz}, 3 \mathrm{H}) .{ }^{13} \mathrm{C}$ NMR $\left(100 \mathrm{MHz}, \mathrm{CDCl}_{3}\right) \delta: 203.4,171.5,143.9,137.2$, 131.6, 130.6, 130.6, 129.1, 127.8, 125.8, 123.9, 45.3, 18.1, 14.5; HRMS (ESI) calcd for $\mathrm{C}_{16} \mathrm{H}_{17} \mathrm{~N}_{2} \mathrm{O}_{2}[\mathrm{M}+\mathrm{H}]$ 269.1285 , found 269.1288 .

1-(2-Benzoyl-4-methylphenyl)-2-( $p$-tolyl)diazene 1 oxide (3n): ${ }^{[15]}$ yellow liquid (46.9 mg, 71\%). ${ }^{1} \mathrm{H}$ NMR (400 $\left.\mathrm{MHz}, \mathrm{CDCl}_{3}\right) \delta: 8.13(\mathrm{~d}, J=8.4 \mathrm{~Hz}, 1 \mathrm{H}), 7.76(\mathrm{~d}, J=7.4$ $\mathrm{Hz}, 2 \mathrm{H}), 7.65(\mathrm{~d}, J=8.4 \mathrm{~Hz}, 2 \mathrm{H}), 7.47 \sim 7.41(\mathrm{~m}, 2 \mathrm{H})$, 7.34 (t, $J=7.7 \mathrm{~Hz}, 2 \mathrm{H}), 7.29$ (s, 1H), 7.08 (d, $J=8.3 \mathrm{~Hz}$, 
2H), $2.47(\mathrm{~s}, 3 \mathrm{H}), 2.30(\mathrm{~s}, 3 \mathrm{H}) ;{ }^{13} \mathrm{C} \mathrm{NMR}(100 \mathrm{MHz}$, $\left.\mathrm{CDCl}_{3}\right) \delta: 194.7,145.3,142.4,141.3,137.4,135.0,133.3$, 131.3, 129.5, 129.4, 129.1, 128.8, 125.5, 123.5, 21.9, 21.6; HRMS (ESI) calcd for $\mathrm{C}_{21} \mathrm{H}_{19} \mathrm{~N}_{2} \mathrm{O}_{2}[\mathrm{M}+\mathrm{H}]^{+} 331.1441$, found 331.1445 .

1-(2-Benzoyl-4-isopropylphenyl)-2-(4-isopropylphenyl)diazene 1-oxide (3o): ${ }^{[15]}$ yellow liquid $(52.5 \mathrm{mg}$, 68\%). ${ }^{1} \mathrm{H}$ NMR (400 MHz, $\left.\mathrm{CDCl}_{3}\right) \delta: 8.16(\mathrm{~d}, J=8.5 \mathrm{~Hz}$, $1 \mathrm{H}), 7.77$ (d, $J=7.5 \mathrm{~Hz}, 2 \mathrm{H}), 7.67$ (d, $J=8.5 \mathrm{~Hz}, 2 \mathrm{H})$, $7.50 \sim 7.44(\mathrm{~m}, 2 \mathrm{H}), 7.38 \sim 7.33(\mathrm{~m}, 3 \mathrm{H}), 7.13(\mathrm{~d}, J=8.5$ $\mathrm{Hz}, 2 \mathrm{H}), 3.08 \sim 2.97(\mathrm{~m}, 1 \mathrm{H}), 2.91 \sim 2.81(\mathrm{~m}, 1 \mathrm{H}), 1.30(\mathrm{~d}$, $J=6.8 \mathrm{~Hz}, 6 \mathrm{H}), 1.19(\mathrm{~d}, J=6.7 \mathrm{~Hz}, 6 \mathrm{H}) ;{ }^{13} \mathrm{C}$ NMR $(100$ $\left.\mathrm{MHz}, \mathrm{CDCl}_{3}\right) \delta: 194.8,153.1,151.5,145.4,141.6,137.5$, $135.1,133.3,129.2,128.8,128.8,127.1,126.8,125.5$, 123.6, 34.4, 34.4, 24.0; HRMS (ESI) calcd for $\mathrm{C}_{25} \mathrm{H}_{27} \mathrm{~N}_{2} \mathrm{O}_{2}$ $[\mathrm{M}+\mathrm{H}]^{+}$387.2067, found 387.2072.

1-(2-Benzoyl-4-methoxyphenyl)-2-(4-methoxyphenyl)diazene 1-oxide (3p): ${ }^{[15]}$ yellow liquid $(50.7 \mathrm{mg}, 70 \%) .{ }^{1} \mathrm{H}$ NMR (400 MHz, $\left.\mathrm{CDCl}_{3}\right) \delta: 8.11(\mathrm{~d}, J=9.1 \mathrm{~Hz}, 1 \mathrm{H}), 7.75$ $(\mathrm{d}, J=9.0 \mathrm{~Hz}, 2 \mathrm{H}), 7.69 \sim 7.67(\mathrm{~m}, 2 \mathrm{H}), 7.36(\mathrm{t}, J=8.6$ $\mathrm{Hz}, 1 \mathrm{H}), 7.27(\mathrm{~d}, J=2.7 \mathrm{~Hz}, 1 \mathrm{H}), 7.27 \sim 7.16(\mathrm{~m}, 1 \mathrm{H})$, $7.02 \sim 6.99(\mathrm{~m}, 1 \mathrm{H}), 6.84(\mathrm{~d}, J=2.6 \mathrm{~Hz}, 1 \mathrm{H}), 6.68(\mathrm{~d}, J=$ $9.0 \mathrm{~Hz}, 2 \mathrm{H}), 3.78(\mathrm{~s}, 3 \mathrm{H}), 3.69(\mathrm{~s}, 3 \mathrm{H}) ;{ }^{13} \mathrm{C}$ NMR $(100$ $\left.\mathrm{MHz}, \mathrm{CDCl}_{3}\right) \delta: 194.2,161.9,160.8,137.5,137.3,133.3$, 129.1, 128.8, 127.9, 127.7, 125.2, 116.0, 113.9, 56.3, 55.8; HRMS (ESI) calcd for $\mathrm{C}_{21} \mathrm{H}_{19} \mathrm{~N}_{2} \mathrm{O}_{4}[\mathrm{M}+\mathrm{H}]^{+}$363.1339, found 363.1344 .

1-(2-Benzoyl-5-methylphenyl)-2-( $m$-tolyl)diazene 1 oxide (3q): ${ }^{[15]}$ yellow liquid (48.2 mg, 73\%). ${ }^{1} \mathrm{H}$ NMR (400 $\left.\mathrm{MHz}, \mathrm{CDCl}_{3}\right) \delta: 7.98(\mathrm{~s}, 1 \mathrm{H}), 7.75(\mathrm{~d}, J=7.6 \mathrm{~Hz}, 2 \mathrm{H})$, $7.47 \sim 7.40(\mathrm{~m}, 5 \mathrm{H}), 7.34(\mathrm{t}, J=7.6 \mathrm{~Hz}, 2 \mathrm{H}), 7.16(\mathrm{t}, J=$ $8.7 \mathrm{~Hz}, 1 \mathrm{H}), 7.07$ (d, $J=7.5 \mathrm{~Hz}, 1 \mathrm{H}), 2.52(\mathrm{~s}, 3 \mathrm{H}), 2.27$ (s, $3 \mathrm{H}) ;{ }^{13} \mathrm{C}$ NMR $\left(100 \mathrm{MHz}, \mathrm{CDCl}_{3}\right) \delta: 194.5,147.8,143.6$, $141.7,138.5,137.6,133.2,132.2,130.9,129.3,129.1$, 128.8, 128.6, 125.6, 124.0, 122.3, 21.7, 21.6; HRMS (ESI) calcd for $\mathrm{C}_{21} \mathrm{H}_{19} \mathrm{~N}_{2} \mathrm{O}_{2}[\mathrm{M}+\mathrm{H}]{ }^{+}$331.1441, found 331.1449 .

1-(2-Benzoyl-6-methylphenyl)-2-(o-tolyl)diazene 1 oxide (3r) $:^{[15]}$ yellow liquid (46.1 mg, 70\%). ${ }^{1} \mathrm{H}$ NMR (400 $\left.\mathrm{MHz}, \mathrm{CDCl}_{3}\right) \delta: 7.79(\mathrm{~d}, J=7.5 \mathrm{~Hz}, 2 \mathrm{H}), 7.64(\mathrm{~d}, J=8.0$ $\mathrm{Hz}, 1 \mathrm{H}), 7.53 \sim 7.44(\mathrm{~m}, 3 \mathrm{H}), 7.42 \sim 7.33(\mathrm{~m}, 3 \mathrm{H}), 7.20 \sim$ $7.13(\mathrm{~m}, 2 \mathrm{H}), 7.07(\mathrm{t}, J=4.6 \mathrm{~Hz}, 1 \mathrm{H}), 2.54(\mathrm{~s}, 3 \mathrm{H}), 2.22(\mathrm{~s}$, $3 \mathrm{H}) ;{ }^{13} \mathrm{C}$ NMR $\left(100 \mathrm{MHz}, \mathrm{CDCl}_{3}\right) \delta: 194.5,148.2,142.4$, $136.9,135.4,134.4,134.2,133.7,132.3,130.9,130.1$, $129.5,129.3,128.7,127.6,126.0,121.5,18.7,18.4$; HRMS (ESI) calcd for $\mathrm{C}_{21} \mathrm{H}_{19} \mathrm{~N}_{2} \mathrm{O}_{2}[\mathrm{M}+\mathrm{H}]^{+} 331.1441$, found 331.1447.

1-(2-Benzoyl-4-fluorophenyl)-2-(4-fluorophenyl)diazene 1-oxide (3s): ${ }^{[15]}$ yellow liquid $(21.6 \mathrm{mg}, 32 \%) .{ }^{1} \mathrm{H}$ NMR $\left(400 \mathrm{MHz}, \mathrm{CDCl}_{3}\right) \delta: 8.27(\mathrm{dd}, J=8.1,3.4 \mathrm{~Hz}, 1 \mathrm{H})$, $7.81 \sim 7.75(\mathrm{~m}, 4 \mathrm{H}), 7.50(\mathrm{~d}, J=7.27 \mathrm{~Hz}, 1 \mathrm{H}), 7.40 \sim 7.31$ $(\mathrm{m}, 3 \mathrm{H}), 7.20(\mathrm{dd}, J=5.2,2.5 \mathrm{~Hz}, 1 \mathrm{H}), 6.97(\mathrm{t}, J=8.9 \mathrm{~Hz}$, $2 \mathrm{H}) ;{ }^{13} \mathrm{C}$ NMR (100 MHz, $\left.\mathrm{CDCl}_{3}\right) \delta$ : 192.7, 164.1 (d, $\left.J_{\mathrm{C}-\mathrm{F}}=254.3 \mathrm{~Hz}\right), 163.1\left(\mathrm{~d}, J_{\mathrm{C}-\mathrm{F}}=251.7 \mathrm{~Hz}\right), 143.4,139.8$ $\left(\mathrm{d}, J_{\mathrm{C}-\mathrm{F}}=3.1 \mathrm{~Hz}\right), 137.4\left(\mathrm{~d}, J_{\mathrm{C}-\mathrm{F}}=3.5 \mathrm{~Hz}\right), 136.6,133.8$, $129.1\left(\mathrm{~d}, J_{\mathrm{C}-\mathrm{F}}=11.6 \mathrm{~Hz}\right), 127.9\left(\mathrm{~d}, J_{\mathrm{C}-\mathrm{F}}=8.5 \mathrm{~Hz}\right), 126.1(\mathrm{~d}$, $\left.J_{\mathrm{C}-\mathrm{F}}=8.9 \mathrm{~Hz}\right), 117.7\left(\mathrm{~d}, J_{\mathrm{C}-\mathrm{F}}=23.0 \mathrm{~Hz}\right), 116.3\left(\mathrm{~d}, J_{\mathrm{C}-\mathrm{F}}=\right.$
$24.6 \mathrm{~Hz}), 115.9\left(\mathrm{~d}, J_{\mathrm{C}-\mathrm{F}}=22.3 \mathrm{~Hz}\right.$ ); HRMS (ESI) calcd for $\mathrm{C}_{19} \mathrm{H}_{13} \mathrm{~F}_{2} \mathrm{~N}_{2} \mathrm{O}_{2}[\mathrm{M}+\mathrm{H}]^{+}$339.0940, found 339.0943.

1-(2-Benzoyl-5-bromophenyl)-2-(3-bromophenyl)diazene 1-oxide (3t): yellow liquid $(30.2 \mathrm{mg}, 33 \%) .{ }^{1} \mathrm{H}$ NMR (400 MHz, $\left.\mathrm{CDCl}_{3}\right) \delta: 8.37(\mathrm{~d}, J=1.69 \mathrm{~Hz}, 1 \mathrm{H}), 7.88$ $(\mathrm{d}, J=1.82 \mathrm{~Hz}, 1 \mathrm{H}), 7.82 \sim 7.80(\mathrm{~m}, 1 \mathrm{H}), 7.75(\mathrm{~d}, J=7.8$ $\mathrm{Hz}, 2 \mathrm{H}), 7.57 \sim 7.55(\mathrm{~m}, 1 \mathrm{H}), 7.51(\mathrm{~d}, J=7.4 \mathrm{~Hz}, 1 \mathrm{H})$, $7.43 \sim 7.40(\mathrm{~m}, 4 \mathrm{H}), 7.19(\mathrm{t}, J=7.3 \mathrm{~Hz}, 1 \mathrm{H}) ;{ }^{13} \mathrm{C} \mathrm{NMR}$ $\left(100 \mathrm{MHz}, \mathrm{CDCl}_{3}\right) \delta: 193.3,144.2,136.8,135.0,133.9$, $133.4,130.7,130.6,130.2,129.1,129.1,127.9,127.0$, 124.6, 124.3, 122.5; HRMS (ESI) calcd for $\mathrm{C}_{19} \mathrm{H}_{13} \mathrm{Br}_{2} \mathrm{~N}_{2} \mathrm{O}_{2}$ $[\mathrm{M}+\mathrm{H}]^{+}$458.9338, found 458.9336 .

Supporting Information The spectroscopic characterization of the products $\mathbf{3 a} \sim \mathbf{3 t}$, X-ray crystallographic data of dimer Pd complex A and GC-MS spectra of Scheme 2c. The Supporting Information is available free of charge via the Internet at http://sioc-journal.cn/.

\section{References}

[1] (a) Surburg, H.; Panten, J. Common Fragrance and Flavour Materials, Wiley Online Library, 2006.

(b) McGrath, N. A.; Brichacek, M.; Njardarson, J. T. J. Chem. Educ. 2010, 87, 1348.

[2] (a) Olah, G. A. Friedel-Crafts Chemistry, Wiley, New York, 1973. (b) Sartori, G.; Maggi, R. Chem. Rev. 2006, 106, 1077.

(c) Fernandez, M.; Tojo, G. In Oxidation of Alcohols to Aldehydes and Ketones: A Guideto Current Common Practice, Ed.: Tojo, E., Springer, New York, 2006.

(d) Sartori, G.; Maggi, R. Advances in Friedel-Crafts Acylation Reactions, CRC Press, Taylor \& Francis Group, 2010.

[3] (a) Moore, E. J.; Pretzer, W. R.; O’Connell, T. J.; Harris, J.; LaBounty, L.; Chou, L.; Grimmer, S. S. J. Am. Chem. Soc. 1992, 114, 5888 .

(b) Chatani, N.; Fukuyama, T.; Kakiuchi, F.; Murai, S. J. Am. Chem. Soc. 1996, 118, 493

(c) Fukuyama, T.; Chatani, N.; Kakiuchi, F.; Murai, S. J. Org. Chem. 1997, 62, 5647.

(d) Chatani, N.; Ie, Y.; Kakiuchi, F.; Murai, S. J. Org. Chem. 1997, 62, 2604.

(e) Ie, Y.; Chatani, N.; Ogo, T.; Marshall, D. R.; Fukuyama, T.; Kakiuchi, F.; Murai, S. J. Org. Chem. 2000, 65, 1475.

[4] Moore, E. J.; Pretzer, W. R.; OConnell, T. J.; Harris, J.; LaBounty, L.; Chou, L.; Grimme, S. S. J. Am. Chem. Soc. 1992, 114, 5888.

[5] Jia, X. F.; Zhang, S. H.; Wang, W. H.; Luo, F.; Cheng, J. Org. Lett. 2009, 11, 3120.

[6] (a) Xiao, F. X.; Shuai, Q.; Zhao, F.; Basle, O.; Deng, G. J.; Li, C. J. Org. Lett. 2011, 13, 1614.

(b) Xu, Z. P.; Xiang, B.; Sun, P. P. RSC Adv. 2013, 3, 1679.

(c) Khemnar, A. B.; Bhanage, B. M. Eur. J. Org. Chem. 2014, 6746. (d) Kishore, R.; Kantam, M. L.; Yadav, J.; Sudhakar, M.; Laha, S.; Venugopal, A. J. Mol. Catal. A: Chem. 2013, 379, 213.

(e) Zhang, Q.; Yang, F.; Wu, Y. J. Chem. Commun. 2013, 49, 6837. (f) Li, M. Z.; Ge, H. B. Org. Lett. 2010, 12, 3464.

[7] (a) Han, S.; Sharma, S.; Park, J.; Kim, M.; Shin, Y.; Mishra, N. K.; Bae, J. J.; Kwak, J. H.; Jung, Y. H.; Kim, I. S. J. Org. Chem. 2014, 79,275

(b) Sharma, S.; Kim, M.; Park, J.; Kim, M.; Kwak, J. H.; Jung, Y. H.; Oh, J. S.; Lee, Y.; Kim, I. S. Eur. J. Org. Chem. 2013, 6656.

[8] Wu, Y. N.; Feng, L. J.; Lu, X.; Kwong, F. Y.; Luo, H. B. Chem. Commun. 2014, 50, 15352.

[9] (a) Weng, J. Q.; Yu, Z. Q.; Liu, X. H.; Zhang, G. F. Tetrahedron Lett. 2013, 54, 1205.

(b) Fang, P.; Li, M. Z.; Ge, H. B. J. Am. Chem. Soc. 2010, 132, 
11898.

(c) Yin, Z. W.; Sun, P. P. J. Org. Chem. 2012, 77, 11339.

(d) Li, C. L.; Wang, L.; Li, P. H.; Zhou, W. Chem.-Eur. J. 2011, 17, 10208.

(e) Wu, Y. N.; Choy, P. Y.; Mao, F.; Kwong, F. Y. Chem. Commun. 2013, 49, 689 .

[10] Yang, Y. Z.; Chen, L.; Zhang, Z. G.; Zhang, Y. H. Org. Lett. 2011, 13,1342 .

[11] (a) Song, H. Y.; Chen, D.; Pi, C.; Cui, X. L.; Wu, Y. J. J. Org. Chem. 2014, 79, 2955.

(b) Li, H. J.; Li, P. H.; Wang, L. Org. Lett. 2013, 15, 620.

(c) Li, H. J.; Li, P. H.; Tan, H.; Wang, L. Chem.-Eur. J. 2013, 19 , 14432.

(d) Li, Z. Y.; Li, D. D.; Wang, G. W. J. Org. Chem. 2013, 78, 10414. (e) Xiong, F.; Qian, C.; Lin, D. G.; Zeng, W.; Lu, X. X. Org. Lett. 2013, 15, 5444 .

[12] Zhao, J. C.; Fang, H.; Xie, C.; Han, J. L.; Li, G. G.; Pan, Y. Asian J. Org. Chem. 2013, 2, 1044.

[13] (a) Ikeda, T.; Tsu, O. Science 1995, 268, 1873.

(b) Kimura, K.; Suzuki, T.; Yokoyama, M. J. Phys. Chem. 1990, 94, 6090

(c) Campbell, D.; Dix, L. R.; Rostron, P. Dyes Pigm. 1995, 29, 77.

(d) Huang, J. M.; Kuo, J. F.; Chen, C. Y. J. Appl. Polym. Sci. 1995, $55,1217$.

(e) Lee, H. K.; Kanazawa, A.; Shiono, T.; Ikeda, T.; Fujisawa, T.; Aizawa, M.; Lee, B. Chem. Mater. 1998, 10, 1402.

(f) Li, H. J.; Li, P. H.; Zhao, Q.; Wang, L. Chem. Commun. 2013, 49, 9170.

[14] For selected examples, see: (a) Hou, Z.; Fujiware, Y.; Taniguchi, H. J. Org. Chem. 1988, 53, 3118.

(b) Sakai, N.; Fuji, K.; Nabeshima, S.; Ikeda, R.; Konakahara, T. Chem. Commun. 2010, 46, 3173.

(c) Wada, S.; Urano, M.; Suzuki, H. J. Org. Chem. 2002, 67, 8254.

(d) Wang, Y.; Cheng, G. L.; Cui, X. L. Chin. J. Org. Chem. 2012, 32, 2018 (in Chinese).

(王勇, 程国林, 崔秀灵, 有机化学, 2012, 32, 2018.)

[15] (a) Sun, M.; Hou, L. K.; Chen, X. X.; Yang, X. J.; Sun, W.; Zang, Y. S. Adv. Synth. Catal. 2014, 356, 3789 (b) Li, H. J.; Li, P. H.; Zhao, Q.; Wang, L. Chem. Commun. 2013 49, 9170.

(c) Yi, M. L.; Cui, X. L.; Zhu, C. W.; Pi, C.; Zhu, W. M.; Wu, Y. J. Asian J. Org. Chem. 2015, 4, 38.

(d) Hou, L. K.; Chen, X. X.; Li, S.; Cai, S. X.; Zhao, Y. X.; Sun, M.; Yang, X. J. Org. Biomol. Chem., 2015, 13, 4160.

[16] (a) Yang, J.; Fu, T.; Long, Y.; Zhou, X. G. Chin. J. Org. Chem. 2017, 37, 1111 (in Chinese).

(杨军, 付婷, 龙洋, 周向葛, 有机化学, 2017, 37, 1111.)

(b) Zhou, Z.; Duan, J. F.; Mu, X. J.; Xiao, S. Y. Chin. J. Org. Chem. 2018, 38, 585 (in Chinese).

(周眰, 段建凤，穆小静，肖尚友，有机化学， 2018, 38, 585.)

(c) Qin, H. F.; Li, X. R. Chin. J. Org. Chem. 1992, 12, 309 (in Chinese).

(秦合法, 李萱荣, 有机化学, 1992, 12, 309.)

[17] (a) Szabó, F.; Daru, J.; Simkó, D.; Nagy, T. Z.; Stirling, A.; Novák, Z. Adv. Synth. Catal. 2013, 355, 685 .

(b) Szabó, F.; Simkó, D.; Novák, Z. RSC Adv. 2014, 4, 3883.

(c) Xiao, F. H.; Chen, S. Q.; Huang, H. W.; Deng, G. J. Eur. J. Org. Chem. 2015, 7919.

[18] Zhang, D.; Cui, X. L.; Yang, F. F.; Q Zhang, Q. Q.; Zhu, Y.; Wu, Y. J. Org. Chem. Front. 2015, 2, 951.

[19] (a) Rosewall, C. F.; Sibbald, P. A.; Liskin, D. V.; Michael, F. E. J. Am. Chem. Soc. 2009, 131, 9488

(b) Xu, L. M.; Li, B. J.; Yang, Z.; Shi, Z. J. Chem. Soc. Rev. 2010, 39,712 .

(c) Sibbald, P. A.; Rosewall, C. F.; Swartz, R. D.; Michael, F. E. J. Am. Chem. Soc. 2009, 131, 15945 .

(d) Powers, D. C.; Ritter, T. Nat. Chem. 2009, 1, 302

(e) Powers, D. C.; Geibel, M. A. L.; Klein, J. E. M. N.; Ritter, T. J. Am. Chem. Soc. 2009, 131, 17050 .

(f) Deprez, N. R.; Sanford, M. S. J. Am. Chem. Soc. 2009, 131, 11234.

(g) Racowski, J. M.; Dick, A. R.; Sanford, M. S. J. Am. Chem. Soc. 2009, 131, 10974.

[20] Christin, G.; Beate, P.; Elisabeth, I.; Karola, R. B. Synthesis 2008 1889. 